\title{
Yoga Reduces Prenatal Depression Symptoms
}

\author{
Jennifer Mitchell ${ }^{1}$, Tiffany Field ${ }^{1,2^{*}}$, Miguel Diego ${ }^{2}$, Debra Bendell ${ }^{1}$, Rae Newton ${ }^{1}$, \\ Martha Pelaez ${ }^{3}$ \\ ${ }^{1}$ Fielding Graduate University, Santa Barbara, USA \\ ${ }^{2}$ Touch Research Institute, University of Miami Medical School, Miami, USA \\ ${ }^{3}$ Florida International University, Miami, USA \\ Email: "tfield@med.miami.edu
}

Received June $28^{\text {th }}, 2012$; revised July $29^{\text {th }}, 2012$; accepted September $1^{\text {st }}, 2012$

\begin{abstract}
This research assessed the effects of yoga on prenatal depression symptoms using archival data. Depressed pregnant women were randomly assigned to either a yoga treatment group $(n=12)$ or a parenting education control group $(\mathrm{n}=12)$. Women in the yoga group participated in classes two times a week for a period of 12 weeks. The attention control group received 12 parenting education sessions on the same schedule. The yoga versus control group showed greater decreases on the depressed affect and somatic/ vegetative subscales and the summary score of the Center for Epidemiological Studies Depression Scale. Thus, yoga appears to reduce depression symptoms in pregnant women.
\end{abstract}

Keywords: Yoga; Prenatal Depression Symptoms

\section{Introduction}

Estimates of the prevalence of prenatal depression span from $11 \%$ (Frisch \& Riecher-Rössler, 2010) to 26\% (Kim et al., 2006). Sub-clinical levels of depression have been noted in as many as $20 \%$ to $49 \%$ of pregnant women (Orr, Blazer, \& James, 2006). Ethnic minorities are considered a higher risk group (Gavin, Melville, Rue, Guo, Dina, \& Katon, 2011), with approximately $25 \%$ of women reporting clinical levels of depresssive symptomatology (Edge, 2007). In addition, a number of psychosocial factors can increase a woman's relative risk, including having a low income and being unmarried (Lancaster, Gold, Flynn, Yoo, Marcus, \& Davis, 2010; Vesga-Lopez, Blanco, Keyes, Olfson, Grant, \& Hasin, 2008). Prenatal depression may also go unreported by women and be overlooked by doctors due to the overlap of symptoms with common physical complaints of pregnancy (Melville, Gavin, Guo, Fan, \& Katon, 2010).

Prenatal depression affects both mother and child. One of the most serious outcomes is premature birth, and prenatal depresssion is considered a serious risk factor for prematurity (Dayan et al., 2006; Field, Diego, Hernandez-Reif, Deeds, Holder et al., 2009; Orr et al., 2006). Prematurity accounted for $12 \%$ of births in the United States in 2008, reflecting an increasing trend since 1981 (Martin, Osterman, \& Sutton, 2010). Most notably, prematurity is the primary reason for infant mortality and is associated with a number of adverse health and development risks (Center for Healthcare Research \& Transformation, 2010; Field, Diego, Dieter et al., 2004).

Prenatal depression can also have long-term effects on the development of the child.

Prenatally depressed mothers have delivered infants who had higher cortisol levels and lower dopamine and serotonin levels, as well as lower birthweight and gestational ages (Field, Diego, Dieter et al., 2004). Negative birth and early childhood out-

\footnotetext{
"Corresponding author.
}

comes associated with prenantal depression include diminished responsivity to stimulation (Field, Diego, \& Hernandez-Reif, 2009; Field, Hernandez-Reif, \& Diego, 2011), and poorer performance on neonatal assessments (Field, Diego, \& HernandezReif, 2010). One area of particular concern is the decreased responsivity by both mother and child within the first six months, which can lead to increased infant distress (Field, 2011a). These negative interactions between mother and child early in life can have serious implications for attachment (Weinfield, Sroufe, Egeland, \& Carlson, 2008). Additional negative effects of prenatal depression on child development include disrupted sleep patterns (Field, Diego, Dieter et al., 2004), behavior problems (de Bruijn, van Bakel, \& van Baar, 2009), developmental delays (Deave, Heron, Evans, \& Emond, 2008), and increased violence in adolescents (Hay, Pawlby, Waters, Perra, \& Sharp, 2010). Moderate pressure massage therapy has decreased prenatal depression (Field, Diego, Hernandez-Reif, Deeds, \& Figueiredo, 2009; Field, Diego, Hernandez-Reif, Schanberg, \& Kuhn, 2004; Field, Hernandez-Reif, Hart et al., 1999) and premature delivery (Field, Diego, Hernandez-Reif, Schanberg, \& Kuhn, 2004; Field, Hernandez-Reif, Hart et al., 1999).

Yoga is another potential alternative therapy that, like massage, is considered safe for pregnant women. Both yoga and massage therapy stimulate pressure receptors which leads to increased vagal activity, so it is not surprising that they also have similar benefits, including decreased depression (Field, 2011b). For these reasons yoga has been considered a form of self-massage, as in the "rubbing of limbs against each other and against the floor" (Field, 2011b). Although yoga decreases depression, research has not yet demonstrated whether yoga decreases depression symptoms in pregnant women (Field, 2011b).

Research on yoga with non-depressed samples has suggested a number of positive outcomes, including decreased anxiety, pain, and premature births (Battle, Uebelacker, Howard, \& Castaneda, 2010; Narendran, Nagarathna, Narendran, Gunasheela, \& Nagendra, 2005). Previous studies with prenatal depression have used yoga interventions that combined breathing, medita- 
tion, and poses. As a result, it is not possible to determine whether the actual poses are therapeutic, or if the changes are related to other aspects of yoga, including meditation and breathing exercises. The current study addressed this gap in the literature and determined whether the actual yoga poses could reduce prenatal depression symptoms. Specifically, our hypothesis was that participation in yoga would result in decreased depression symptoms, as measured by depressed affect and somatic/vegetative symptom scores on the Center for Epidemiological Studies-Depression scale (CES-D; Radloff, 1977). To test this hypothesis, a yoga group was compared to an attention comtrol group.

Domains typically associated with depressive symptomatology and assessed by the subscales of the CES-D include positive and depressed affect, somatic/vegetative signs, and interpersonal distress. Research on the effects of yoga on vagal activity suggests that decreases would occur specifically on the depressed affect and somatic/vegetative signs subscales following yoga. The basis for this hypothesis was that vagal activity has increased following yoga (Sathyaprabha et al., 2008). Since vagal activity stimulates the muscles that control facial expressions and vocal intonation (Porges, 2001), it could be argued that increased vagal activity in depressed pregnant women would lead to decreased depressed affect (Porges, 2001). Vagal activity has also been linked to the regulation of somatic/vegetative signs including appetite and sleep, whereby lower vagal activity was associated with eating and sleeping problems (Bodenlos et al., 2007; El-Sheikh et al., 2007). Thus, depressed affect and vegetative state/somatic complaints were expected to decrease following yoga.

\section{Methods}

\section{Participants}

The sample was comprised of 24 participants from a pilot study conducted for a larger ongoing study on yoga effects on prenatal depression. Participants were recruited from two prenatal ultrasound clinics affiliated with a large private university medical center in the South. The 24 participants were clinically depressed pregnant women who were randomly assigned to either the yoga treatment group $(n=12)$ or parenting education attention control group $(n=12)$. The recruitment criteria were: 1) meeting diagnostic criteria for depression on the Structured Clinical Interview for Depression (SCID); 2) being pregnant with one child; 3) having an uncomplicated pregnancy with no medical illness; 4) being younger than 40-years-old; 5) not having a co-morbid mental health issue; and 6) not using drugs (i.e., prescribed or illicit) that could affect cortisol levels. Previous samples recruited from these clinics had a very low incidence $(3 \%-5 \%)$ of treatment for prenatal depression (i.e., psychotherapy or antidepressants), so these were not exclusion criteria.

The sample comprised women ranging in age from 18- to $37-$ years-old, with an average age of 26.6 years. The participants were primarily low-income minorities who had a high school education. Table 1 provides aggregate information on the participants' ethnicity, SES, and level of education.

\section{Measures}

The CES-D (Radloff, 1977) was administered at the beginning $(\mathrm{M}=20$ weeks gestation $)$ and at the end of the treatment period $(\mathrm{M}=32$ weeks gestation). The CES-D is a 20-item self-report measure that assesses frequency of current depres- sion symptoms over the past week (Radloff, 1977). Targeted symptoms include "depressed mood, feelings of guilt and worthlessness, feelings of helplessness and hopelessness, loss of energy, and disturbances of sleep and appetite" (Radloff \& Teri, 1986). Likert frequency ratings include most of the time (6 - 7 days), occasionally, (3 - 4 days), some of the time (1 - 2 days), and rarely (less than a day). Individuals are asked to rate each item from 0 to 3 based on how often they have felt this way, with higher scores indicating greater frequency. Total summary scores range from 0 to 60 , with clinical levels of depressive symptomatology associated with scores of 16 or higher (Radloff, 1977). Subscale scores are provided for depressed affect, positive affect, somatic/vegetative signs, and interpersonal distress (see Table 2; Radloff \& Teri, 1986). The CES-D has acceptable validity and reliability for various demographic

Table 1.

Demographic characteristics of the sample.

\begin{tabular}{ccc}
\hline Characteristic & Frequency & Percentage \\
\hline Ethnicity & & \\
Black & 14 & 58.3 \\
Hispanic & 6 & 25.0 \\
White & 2 & 8.3 \\
Other & 2 & 8.4 \\
SES & & \\
Middle & 2 & 8.4 \\
Lower-Middle & 5 & 20.8 \\
Lower-Lower & 17 & 70.8 \\
Level of Education & & \\
7 - 9 Years & 1 & 4.2 \\
10 - 11 Years & 5 & 20.8 \\
High School Graduate & 10 & 41.7 \\
1 - 3 Years of College & 8 & 33.3 \\
\hline
\end{tabular}

Table 2.

CES-D items by subscale.

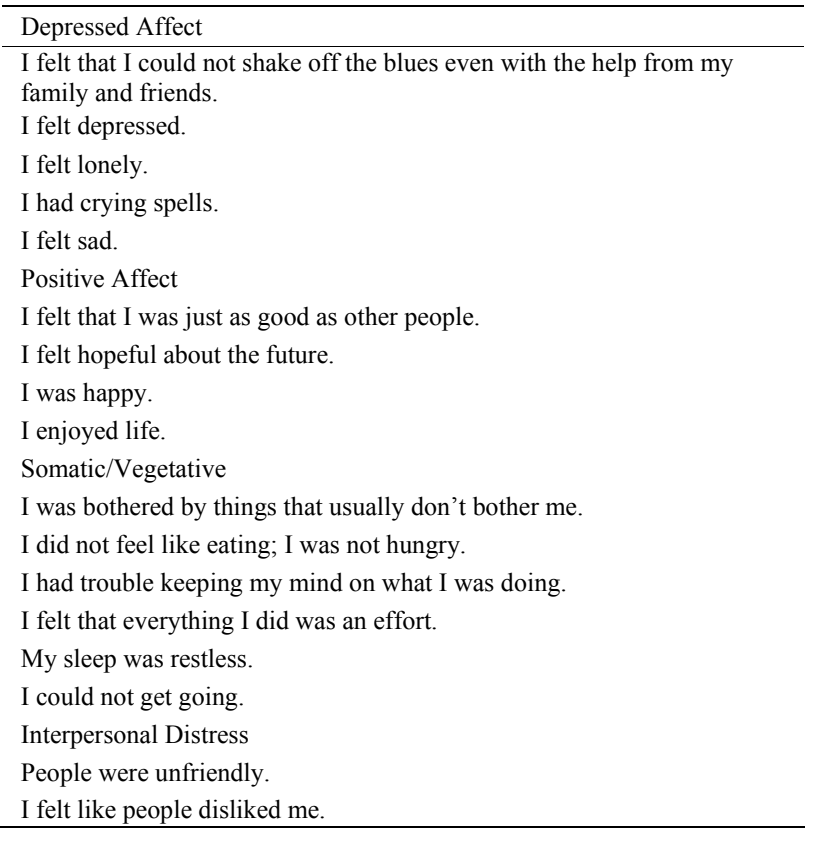

Note: Items 4, 8, 12, and 16 are reverse scored. 
variables including geographic location, level of education, age, ethnicity (black, white, Hispanic, Asian, and European), and language (Radloff \& Teri, 1986).

The CES-D is a valid and reliable measure of depression symptoms, and a precise tool for assessing symptom changes across time (Weissman, Sholomskas, Pottenger, Prusoff, \& Locke, 1977). Radloff (1977) reported internal consistency of .85 for the general population and .90 for clinical samples, moderate test-retest reliability correlations between .51 and .67 over 8 weeks, and split-half reliabilities of .77 to .92 . In a study on black, white, and Mexican-American ethnicities, no differences were noted in internal consistency and reliability scores among the groups (Roberts, 1980). Among low income, minority women, the CES-D had moderate criterion validity and was related to an MDD diagnosis (Thomas, Jones, Scarinci, Mehan, $\&$ Brantley, 2001). A similar study on low SES minorities supported the four-factor model (i.e., depressed affect, positive affect, somatic/vegetative complaints, and interpersonal problems) originally observed in the general population (Nguyen, Kitner-Triolo, Evans, \& Zonderman, 2004).

Finally, a study on a high-risk sample of women with prenatal depression supported these early findings, with internal consistency (Cronbach alpha) ranging from .88 to .93 (Maloni, Park, Anthony, \& Musil, 2005). Additionally, test-retest reliability indicated stable results over time (i.e., admission, 2-weeks, and 4 -weeks), as well as statistically significant $(p>.01)$ convergent validity with other depression symptoms scales (Maloni et al., 2005). CES-D scores were also sensitive to decreases in depression symptoms resulting from massage therapy (Field, Diego, Hernandez-Reif, Schanberg, \& Kuhn, 2004; Field, Hernandez-Reif, Hart et al., 1999). Together, these findings suggested that the CES-D was an appropriate measure of depression symptoms for this study's sample and design.

\section{Procedures}

Women in the yoga group participated in 20-minute sessions two times a week for a period of 12 weeks. A trained yoga instructor led group participants through a routine specifically designed for women in their second and third trimesters of pregnancy (see Table 3 for routine). The control group participated in parenting education sessions to control for the effects of attention and social support received by the women in the yoga group. The yoga and parenting education groups were the same size and followed the same weekly schedule. Participants in both groups were paid $\$ 20$ for each session to compensate for expenses related to lost wages, childcare, and transportation.

\section{Results}

Fisher-Freeman-Halton tests revealed no significant differences between the yoga and control groups on ethnicity $\left(x^{2}=\right.$ $4.20, p=.26)$, socioeconomic status $\left(x^{2}=2.40, p=.46\right)$ or level of education $\left(x^{2}=4.88, p=.11\right)$. In addition, a one-way ANOVA indicated that the groups did not differ on maternal age, $F(1,20)=.05, p=.83$.

Repeated measures ANOVAs were conducted to test for changes in CES-D scores over time for both groups. In these analyses, "group" was designated by treatment or control condition, and "time" was defined by changes from pretest to posttest scores. Table $\mathbf{4}$ presents mean summary and subscale scores for the CES-D by group. A significant repeated measures
Table 3.

Yoga postures.

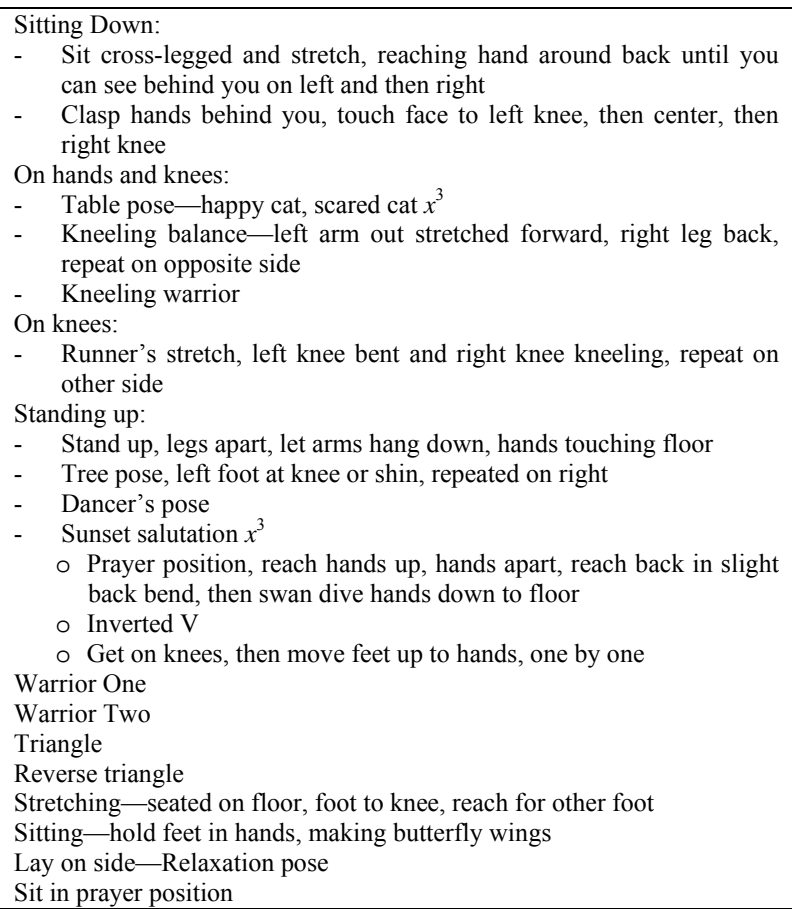

\section{Table 4}

Mean Scores on Pretest and Posttest CES-D Summary and Subscales by Group (Standard deviations in parentheses).

\begin{tabular}{|c|c|c|c|c|}
\hline \multirow{3}{*}{ Scale } & \multicolumn{4}{|c|}{ Group } \\
\hline & \multicolumn{2}{|c|}{ Yoga } & \multicolumn{2}{|c|}{ Control } \\
\hline & Pretest & Posttest & Pretest & Posttest \\
\hline CES-D Summary & $27.50(10.04)$ & $17.92(8.67)$ & $22.42(8.83)$ & $21.42(9.79)$ \\
\hline Depressed Affect & $8.17(4.08)$ & $4.83(3.59)$ & $5.33(3.03)$ & $5.50(3.42)$ \\
\hline Positive Affect & $5.00(2.59)$ & $4.08(1.73)$ & $5.25(4.92)$ & $4.92(2.68)$ \\
\hline Somatic/Vegetative & $9.50(3.23)$ & $6.75(2.73)$ & $7.00(3.81)$ & $7.58(4.01)$ \\
\hline Interpersonal Distress & $1.33(1.23)$ & $.75(1.60)$ & $1.43(1.31)$ & $1.25(1.22)$ \\
\hline
\end{tabular}

effect indicated differences between pretest and posttest CES-D summary scores, $F(1,22)=9.22, p<.01$. Although there was no significant group effect, $F(1,22)=0.54, p=.82$, the group by time interaction effect was significant, $F(1,22)=6.07, p$ $<.05, \eta_{p}^{2}=.22$. A repeated measures by group ANOVA was also conducted for each of the CES-D subscales. Group by time interaction effects were significant for the depressed affect, $F(1$, $22)=7.21, p<.05, \eta_{p}^{2}=.25$ and somatic/vegetative subscales, $F(1,22)=5.98, p<.05, \eta_{p}^{2}=.21$.

Additional analyses with paired sample $\mathrm{t}$ tests were conducted for the CES-D summary and subscale scores. Results indicated that the pretest/posttest difference in CES-D summary scores was significant for the yoga group, but not the control group (see Table 5). Paired sample t tests for the pretest/posttest differences in subscale scores are presented in Table 5. No significant differences were found for the control group subscale scores. Additionally, no significant differences were found for the positive affect and interpersonal distress subscales in the yoga group. However, the differences in pre- and post-test scores on the depressed affect and somatic/vegetative subscales were statistically significant for the yoga group. 
Table 5.

Change in pretest/posttest CES-D summary scale and subscales by group (standard deviations in parentheses).

\begin{tabular}{lcccr}
\hline \multirow{2}{*}{ Scale } & \multicolumn{2}{c}{ Yoga } & \multicolumn{2}{c}{ Control } \\
\cline { 2 - 5 } & $M(S D)$ & $t$ & $M(S D)$ & \multicolumn{1}{c}{$t$} \\
\hline CES-D Summary & $-9.58(11.06)$ & $-3.00^{*}$ & $-1.00(4.84)$ & -.72 \\
Depressed Affect & $-3.33(3.96)$ & $-2.91^{*}$ & $.17(2.17)$ & .27 \\
Positive Affect & $-.92(2.61)$ & -1.21 & $-.33(2.46)$ & -.47 \\
Somatic/Vegetative & $-2.75(4.14)$ & $-2.30^{*}$ & $.58(2.27)$ & .89 \\
Interpersonal Distress & $-.58(1.31)$ & -1.54 & $-.17(1.27)$ & -.46 \\
\hline
\end{tabular}

${ }^{*} p<.05$, two-tail.

\section{Discussion}

Massage therapy and yoga are thought to stimulate pressure receptors that result in increased vagal activity and lead to decreased depression (Field, 2011b). Since the mechanisms of action seem to be similar, this study hypothesized that, like massage therapy, yoga would result in decreased prenatal depression.

The results from this study offer support for yoga as another alternative treatment for depressed pregnant women. Yoga not only decreased prenatal depression, but also decreased depressed affect and somatic/vegetative signs. These effects may relate to the increased vagal activity previously noted following yoga (Sathyaprabha et al., 2008). Since vagal activity stimulates the muscles that control facial expressions and vocal intonation (affect) and has been linked to vegetative signs (Porges, 2001), it could mediate the decrease in depressed affect and somatic/vegetative symptom scores for the yoga group. Yoga has the benefits of moderate intensity exercise and relaxation, while also offering improved sleep, fatigue, and stress levels (Ross \& Thomas, 2010). These results, in turn, support the clinical utility of yoga for reducing common complaints of pregnant women that can be exacerbated by prenatal depression. Although the results of this study suggested a statistically greater decrease in prenatal depression for the yoga group, this finding may not be clinically significant. For this study, the mean posttest CES-D summary scores for the yoga and control groups were 17.9 and 21.4, respectively. Although the yoga group exhibited greater symptom reduction, the mean CES-D was still above the clinical cutoff score of 16 . The yoga reduced depressive symptoms to subclinical levels in $55 \%$ of participants compared to $11 \%$ in the control group. However, almost half of the participants in the yoga group still reported clinical levels of depressive symptoms.

Future research using a larger sample size will help to support these preliminary findings. In addition, studies examining the effects of yoga and comparable treatments (e.g., massage or exercise) will elucidate the relative efficacy of yoga in ameliorating prenatal depression. Additional research exploring the mechanisms underlying yoga effects on prenatal depression will provide further support for yoga as a viable treatment. Nonetheless, the current study suggests that yoga poses practiced over a 12-week period during pregnancy can reduce depression symptoms in depressed women.

\section{Acknowledgements}

We would like to thank all the pregnant women who participated in this study. This research was supported by an NIH grant (HD056036), a Senior Research Scientist Award (AT001585) and funding from Johnson and Johnson Pediatric Institute to the Touch Research Institute. Author's correspondence email address is tfield@med.miami.edu

\section{REFERENCES}

Battle, C. L., Uebelacker, L. A., Howard, M., \& Castaneda, M. (2010). Prenatal yoga and depression during pregnancy. Birth, 37, 353-354. doi:10.1111/j.1523-536X.2010.00435 1.X

Bodenlos, J. S., Kose, S., Borckardt, J. J., Nahas, Z., Shaw, D., O’Neil, P. M., \& George, M. S. (2007). Vagus nerve stimulation and emotional responses to food among depressed patients. Journal of Diabetes Science and Technology, 1, 771-779.

Center for Healthcare Research \& Transformation (2010). Prematurity. In Price of care (pp. 1-12). Ann Arbor, MI: Center for Healthcare Research \& Transformation

Dayan, J., Creveuil, C., Marks, M. N., Conroy, S., Herlicoviez, M., Dreyfus, M., \& Tordjman, S. (2006). Prenatal depression, prenatal anxiety, and spontaneous preterm birth: A prospective cohort study among women with early and regular care. Psychosomatic Medicine, 68, 938-946. doi:10.1097/01.psy.0000244025.20549.bd

Edge, D. (2007). Ethnicity, psychosocial risk, and perinatal depression: A comparative study among inner-city women in the United Kingdom. Journal of Psychosomatic Research, 63, 291-295. doi:10.1016/j.jpsychores.2007.02.013

El-Sheikh, M., Erath, S. A., \& Keller, P. S. (2007). Children's sleep and adjustment: The moderating role of vagal regulation. Journal of Sleep Research, 16, 396-405. doi:10.1111/j.1365-2869.2007.00618.x

Essau, C. A., Lewinsohn, P. M., Seeley, J. R., \& Sasagawa, S. (2010). Gender differences in the developmental course of depression. Journal of Affective Disorders, 127, 185-190. doi:10.1016/i.jad.2010.05.016

Field, T. (2011a). Prenatal depression effects on early development: A review. Infant Behavior and Development, 34, 1-14. doi:10.1016/j.infbeh.2010.09.008

Field, T. (2011b). Yoga clinical research review. Complementary Therapies in Clinical Practice, 17, 1-8. doi:10.1016/j.ctcp.2010.09.007

Field, T., Diego, M., Dieter, J., Hernandez-Reif, M., Schanberg, S., Kuhn, C., \& Bendell, D. (2004). Prenatal depression effects on the fetus and the newborn. Infant Behavior \& Development, 27, 216-229. doi:10.1016/j.infbeh.2003.09.010

Field, T., Diego, M., \& Hernandez-Reif, M. (2009). Depressed mothers' infants are less responsive to faces and voices. Infant Behavior and Development, 32, 239-244. doi:10.1016/j.infbeh.2009.03.005

Field, T., Diego, M., \& Hernandez-Reif, M. (2010). Prenatal depression effects and interventions: A review. Infant Behavior and Development, 33, 409-418. doi:10.1016/j.infbeh.2010.04.005

Field, T., Diego, M., Hernandez-Reif, M., Deeds, O., \& Figueiredo, B. (2009). Pregnancy massage reduces prematurity, low birthweight and postpartum depression. Infant Behavior and Development, 32, 454460. doi:10.1016/j.infbeh.2009.07.001

Field, T., Diego, M., Hernandez-Reif, M., Deeds, O., Holder, V., Schanberg, S., \& Kuhn, C. (2009). Depressed pregnant black women have a greater incidence of prematurity and Low birthweight outcomes. Infant Behavior and Development, 32, 10-16. doi:10.1016/j.infbeh.2008.09.005

Field, T., Diego, M., Hernandez-Reif, M., Schanberg, S., \& Kuhn, C. (2004). Massage therapy effects on depressed pregnant women. Journal of Psychosomatic Obstetrics and Gynaecology, 25, 115-122. doi:10.1080/01674820412331282231

Field, T., Hernandez-Reif, M., \& Diego, M. (2011). Depressed mothers' newborns are less responsive to animate and inanimate stimuli. Infant and Child Development, 20, 94-105. doi:10.1002/icd.687

Field, T., Hernandez-Reif, M., Diego, M., Figueiredo, B., Schanberg, S., \& Kuhn, C. (2006). Prenatal cortisol, prematurity and low birthweight. Infant Behavior and Development, 29, 268-275. doi:10.1016/j.infbeh.2005.12.010

Field, T., Hernandez-Reif, M., Hart, S., Theakston, H., Schanberg, S., \& Kuhn, C. (1999). Pregnant women benefit from massage therapy. 
Journal of Psychosomatic Obstetrics and Gynecology, 20, 31-38.

Frisch, U., \& Riecher-Rössler, A. (2010). Depression during pregnancy. Therapeutische Umschau. Revue Thérapeutique, 67, 571-575. doi:10.1024/0040-5930/a000097

Gavin, A. R., Melville, J. L., Rue, T., Guo, Y., Dina, K. T., \& Katon, W. J. (2011). Racial differences in the prevalence of antenatal depression. General Hospital Psychiatry, 33, 87-93. doi:10.1016/j.genhosppsych.2010.11.012

Hay, D. F., Pawlby, S., Waters, C. S., Perra, O., \& Sharp, D. (2010). Mothers' antenatal depression and their children's antisocial outcomes. Child Development, 81, 149-165. doi:10.1111/j.1467-8624.2009.01386.x

Kessler, R. C., Birnbaum, H., Bromet, E., Hwang, I., Sampson, N., \& Shahly, V. (2010). Age differences in major depression: Results from the National Comorbidity Survey Replication (NCS-R). Psychological Medicine, 40, 225-237. doi:10.1017/S0033291709990213

Kim, H. G., Mandell, M., Crandall, C., Kuskowski, M. A., Dieperink, B., \& Buchberger, R. L. (2006). Antenatal psychiatric illness and adequacy of prenatal care in an ethnically diverse inner-city obstetric population. Archives of Women's Mental Health, 9, 103-107. doi:10.1007/s00737-005-0117-5

Lancaster, C. A., Gold, K. J., Flynn, H. A., Yoo, H., Marcus, S. M., \& Davis, M. M. (2010). Risk factors for depressive symptoms during pregnancy: A systematic review. American Journal of Obstetrics and Gynecology, 202, 5-14. doi:10.1016/j.ajog.2009.09.007

Maloni, J. A., Park, S., Anthony, M. K., \& Musil, C. M. (2005). Measurement of antepartum depressive symptoms during high-risk pregnancy. Research in Nursing \& Health, 28, 16-26. doi:10.1002/nur.20051

Martin, J. A., Osterman, M. J. K., \& Sutton, P. D. (2010). Are preterm births on the decline in the United States? Recent data from the National Vital Statistics System. NCHS Data Brief, 39, 1-8.

Melville, J. L., Gavin, A., Guo, Y., Fan, M.-Y., \& Katon, W. J. (2010). Depressive disorders during pregnancy: Prevalence and risk factors in a large urban sample. Obstetrics and Gynecology, 116, 1064-1070. doi:10.1097/AOG.0b013e3181f60b0a

Narendran, S., Nagarathna, R., Narendran, V., Gunasheela, S., \& Nagendra, H. R. R. (2005). Efficacy of yoga on pregnancy outcome. Journal of Alternative and Complementary Medicine, 11, 237-244. doi:10.1089/acm.2005.11.237

Nguyen, H. T., Kitner-Triolo, M., Evans, M. K., \& Zonderman, A. B. (2004). Factorial invariance of the CES-D in low socioeconomic status African Americans compared with a nationally representative sample. Psychiatry Research, 126, 177-187. doi:10.1016/j.psychres.2004.02.004

Orr, S. T., Blazer, D. G., \& James, S. A. (2006). Racial disparities in elevated prenatal depressive symptoms among black and white women in eastern North Carolina. Annals of Epidemiology, 16, 463-468. doi:10.1016/j.annepidem.2005.08.004

Porges, S. W. (2001). The polyvagal theory: Phylogenetic substrates of a social nervous system. International Journal of Psychophysiology: Official Journal of the International Organization of Psychophysiology, 42, 123-146. doi:10.1016/S0167-8760(01)00162-3

Radloff, L. (1977). The CES-D scale: A self-report depression scale for research in the general population. Applied Psychological Methods, 1, 385-401. doi:10.1177/014662167700100306

Radloff, L., \& Teri, L. (1986). Use of the Center for Epidemiological Studies-Depression Scale with older adults. Clinical Gerontologist, 5, 119-135. doi:10.1300/J018v05n01 06

Roberts, R. E. (1980). Reliability of the CES-D scale in different ethnic contexts. Psychiatry Research, 2, 125-134. doi:10.1016/0165-1781(80)90069-4

Ross, A., \& Thomas, S. (2010). The health benefits of yoga and exercise: A review of comparison studies. Journal of Alternative and Complementary Medicine, 16, 3-12. doi:10.1089/acm.2009.0044

Thomas, J. L., Jones, G. N., Scarinci, I. C., Mehan, D. J, \& Brantley, P. J. (2001). The utility of the CES-D as a depression screening measure among low-income women attending primary care clinics. International Journal of Psychiatry in Medicine, 31, 25-40. doi:10.2190/FUFR-PK9F-6U10-JXRK

Vesga-Lopez, O., Blanco, C., Keyes, K., Olfson, M., Grant, B. F., \& Hasin, D. S. (2008). Psychiatric disorders in pregnant and postpartum women in the United States. Archives of General Psychiatry, 65, 805-815. doi:10.1001/archpsyc.65.7.805

Weinfield, N. S., Sroufe, L. A., Egeland, B., \& Carlson, E. (2008). Individual differences in infant-caregiver attachment: Conceptual and empirical aspects of security. In J. Cassidy, \& P. R. Shaver (Eds.), Handbook of attachment: Theory, research, and clinical applications (2nd ed., pp. 78-101). New York: Guilford Press.

Weissman, M. M., Sholomskas, D., Pottenger, M., Prusoff, B. A., \& Locke, B. Z. (1977). Assessing depressive symtoms in five psychiatric populations: A validation study. American Journal of Epidemiology, 106, 203-214. 